Mathematical Modelling and Analysis

Volume 20 Number 6, November 2015, 737-753

http://dx.doi.org/10.3846/13926292.2015.1111266

(c) Vilnius Gediminas Technical University, 2015
Publisher: Taylor\&Francis and VGTU

http://www.tandfonline.com/TMMA

eISSN: $1648-3510$

\title{
A Block by Block Method for Solving System of Volterra Integral Equations with Continuous and Abel Kernels
}

\section{Roghayeh Katani $^{a}$ and Sedaghat Shahmorad ${ }^{b}$}

a Faculty of sciences, University of Yasouj

Yasouj, Iran.

${ }^{b}$ Faculty of mathematical sciences, University of Tabriz

Tabriz, Iran

E-mail(corresp.): katani@yu.ac.ir

E-mail: shahmorad@tabrizu.ac.ir

Received August 18, 2014; revised October 8, 2015; published online November 15, 2015

\begin{abstract}
The aim of the present paper is to introduce a block by block method for solving system of nonlinear Volterra integral equations with continuous kernels and system of Abel integral equations. We prove convergence of the method and show that its convergence order is at least six. To illustrate performance of the method, numerical experiments are presented and they are compared with HPM (Homotopy Perturbation Method) and RBFN (Radial Basis Function Network) method. The given results demonstrate remarkable ability of the proposed method.
\end{abstract}

Keywords: Abel integral equations, Romberg quadrature rule, block by block method, nonlinear Volterra integral equations.

AMS Subject Classification: 65R20.

\section{Introduction}

Consider a system of Volterra integral equations (VIEs) of the form

$$
\mathbf{f}(x)=\mathbf{g}(x)+\int_{0}^{x}(\mathbf{K}(x, s, \mathbf{f}(s)) \mathbf{H}(x, s)) d s, \quad 0 \leq s \leq x \leq X,
$$

where $\mathbf{f}, \mathbf{g}:[0, X] \longrightarrow \mathbb{R}^{n}$ and $\mathbf{K}(x, s, f):[0, X] \times[0, X] \times \mathbb{R}^{n} \longrightarrow \mathbb{R}^{n \times n}$ are given continuous functions and $\mathbf{H}:[0, X] \times[0, X] \longrightarrow \mathbb{R}^{n}$ is defined by $\mathbf{H}=\left[H_{1}(x, s), \ldots, H_{n}(x, s)\right]^{T}$, with $H_{j}(x, s)=1, j=1,2, \ldots, n$ for the equations with continuous kernels and

$$
H_{j}(x, s) \equiv \frac{1}{|x-s|^{\alpha_{j}}}, \quad 0<\alpha_{j}<1, \quad j=1,2, \ldots, n
$$

for the Abel equations. 
There are many physical and biological problems which are modeled as VIEs with weakly singular kernels such as reaction-diffusion problems [9], the behavior of viscoelastic materials in mechanics, superfluidity problems, propagation of a flame, soft tissues like mitral valves of the aorta in the human heart (see [6]). A system of Abel-VIEs may arise either from semi-discretization along space of partial differential equations of fractional order, which models for example some anomalous diffusion and sub-diffusion processes [15] or arises from semi-discretization of Volterra-Fredholm integral equations with singular kernels, some of which occurs in the modeling of coding mechanism in the transmission of nervous signals among neurons [7].

There are also many numerical methods for solving system of VIEs, which some of them are briefly described as follows:

The Chebyshev collocation method has been presented in [2] to solve a system of linear integral equations in terms of Chebyshev polynomials. This method transforms the integral system to the corresponding matrix equation by use of Chebyshev collocation points which is solved for the Chebyshev coefficients. Golbabai et al. [8] proposed a novel learning strategy for radial basis function networks (RBFN). By adjusting the parameters of the hidden layer, including the RBF centers and widths, the weights of the output layer are adapted by local optimization methods. In [18] continuous approximations to the solution of the system of Volterra integral equations of the first and second kinds are sought by methods using spline functions of degree $m$ and deficiency- $(k-1)$, i.e. in $C^{m-k}$. The resulting method is called an $(m, k)$ method. An expansion method in [16] reduces a system of integral equations to the corresponding linear system of ordinary differential equations. After constructing boundary conditions, this system reduces to a system of algebraic equations that can be solved by a direct or iterative method. Maleknejad et al. [13] used operational matrices of piecewise constant orthogonal functions on the interval $[0,1)$ to solve system of singular VIEs of convolution type. They use the Laplace transform and then found numerical inversion of Laplace transform by operational matrices. In this field some new versions of the Adomian Decomposition Method (ADM), Homotopy Perturbation Method (HPM) and Runge Kutta methods were also introduced (see for example $[3,12,20]$ ).

A block by block method is essentially an extrapolation procedure described by Young [19] for the first time. It has the advantages of being self starting and producing a block of values simultaneously. It is also effective for the problems defined in a large interval. In what follows, we propose a new extension of the block by block method for solving system of Volterra integral equations with continuous and Abel kernels. Of course for Abel equations, to note that the results of this paper are valid if $f(x)$ has sufficient continuity, otherwise it would be necessary to develop special starting formula, for this case see $[1,4,5]$.

Our method has the following extra advantages:

1. The order of convergence is at least $O\left(h^{6}\right)$ (it is $O\left(h^{4}\right)$ by using Simpson rule [11]) for a given step size $h$.

2. By increasing number of blocks, the order of convergence increases such that it would be at least $O\left(h^{8}\right)$ and $O\left(h^{10}\right)$ respectively for 8 and 16 blocks. 
3. In the first step of Romberg quadrature rule, the Simpson rule can be used instead of trapezoidal rule, then the order of convergence will be at least $O\left(h^{8}\right)$ for a 4 blocks method.

The rest of the paper is organized as follows. In sections 2 and 3 , the method will be described for the system of VIEs with continuous and Abel kernels respectively and then a convergence result will be proven. Finally, the method will be illustrated by numerical results in section 4 .

\section{System of VIEs with continuous kernels}

Consider a system of Volterra integral equations of the form

$$
\mathbf{f}(x)=\mathbf{g}(x)+\int_{0}^{x} \mathbf{K}(x, s, \mathbf{f}(s)) \mathbf{H}(x, s) d s, \quad 0 \leq s \leq x \leq X
$$

where

$$
\begin{aligned}
& \mathbf{f}(x)=\left[f_{1}(x), f_{2}(x), \ldots, f_{n}(x)\right]^{T}, \quad \mathbf{g}(x)=\left[g_{1}(x), g_{2}(x), \ldots, g_{n}(x)\right]^{T}, \\
& \mathbf{H}(x, s)=[1,1, \ldots, 1]^{T}
\end{aligned}
$$

and

$$
\mathbf{K}(x, s, \mathbf{f}(s))=\left[\begin{array}{ccc}
k_{1,1}(x, s, \mathbf{f}(s)) & \ldots & k_{1, n}(x, s, \mathbf{f}(s)) \\
k_{2,1}(x, s, \mathbf{f}(s)) & \ldots & k_{2, n}(x, s, \mathbf{f}(s)) \\
& \vdots & \\
k_{n, 1}(x, s, \mathbf{f}(s)) & \ldots & k_{n, n}(x, s, \mathbf{f}(s))
\end{array}\right]
$$

\subsection{Existence and uniqueness of solution}

Sufficient conditions for the existence and uniqueness of solution for equation (2.1) are described by the Theorem 1.

Definition 1. For $d>0$, we define $S_{d}^{n}=\left\{\mathbf{f} \in \mathbb{R}^{n}:\|\mathbf{f}\|_{1} \leq d\right\}$, where $\mathbb{R}^{n}$ is the $n$-dimensional Euclidean space and $\|\mathbf{f}\|_{1}=\sum_{i=1}^{n}\left|f_{i}\right|$.

Theorem 1. Consider the system

$$
\mathbf{f}(x)=\mathbf{g}(x)+\int_{0}^{x} \mathbf{K}(x, s, \mathbf{f}(s)) d s, \quad x>0
$$

with

$$
\begin{aligned}
& \mathbf{f}(x)=\left[f_{1}(x), f_{2}(x), \ldots, f_{n}(x)\right]^{T}, \quad \mathbf{g}(x)=\left[g_{1}(x), g_{2}(x), \ldots, g_{n}(x)\right]^{T}, \\
& \mathbf{K}(x, s, \mathbf{f}(s))=\left[k_{1}(x, s, \mathbf{f}(s)), k_{2}(x, s, \mathbf{f}(s)), \ldots, k_{n}(x, s, \mathbf{f}(s))\right]^{T}
\end{aligned}
$$

where $\mathbf{g}$ and $\mathbf{K}$ are continuous respectively on $[0,+\infty)$ and

$$
D:=\left\{(x, s, \mathbf{f}) \mid 0<s<x<\infty, \mathbf{f} \in \mathbb{R}^{n}\right\} .
$$

Suppose that 
(i) for all $X>0$ and the continuous function $\Psi:[0, X] \rightarrow \mathbb{R}^{n}, \mathbf{K}(x, s, \Psi(s))$ $i$ continuous for $s \in(0, x)$, and $\left\|\int_{0}^{x} \mathbf{K}(x, s, \Psi(s)) d s\right\|_{1}<\infty$ for $x \in[0, X]$;

(ii) there exists a continuous function $Q(x, s)$ on $0<s<x<\infty$ satisfying

$$
\int_{0}^{x} Q(x, s) d s<\infty, \quad x \in(0, \infty)
$$

and $\forall X>0$, as $\delta^{+} \rightarrow 0$,

$$
\int_{x}^{x+\delta} Q(x+\delta, s) d s \rightarrow 0, \quad x \in[0, X]
$$

uniformly in $x$, such that

$$
\|\mathbf{K}(x, s, \mathbf{z})-\mathbf{K}(x, s, \mathbf{y})\|_{1} \leq Q(x, s)\|\mathbf{z}-\mathbf{y}\|_{1}
$$

for $\mathbf{z}, \mathbf{y} \in S_{d}^{n}(\forall d>0)$.

Then, there exists an $\alpha>0$, such that the system of Volterra integral equations (2.2) has a unique continuous solution on $[0, \alpha]$. Furthermore, if there exists $B>0$, such that $\|\mathbf{f}(x)\|_{1}$ is bounded by $B$ on all intervals $[0, \beta](\beta>0)$ on which eq. (2.2) has a unique continuous solution, then equation (2.2) has a unique continuous solution on $[0, \infty)$.

Proof. See [10].

\subsection{The quadrature rule}

In this section, we state basic formulation of the Romberg quadrature rule. To recall this, we use the trapezoidal rule for $\int_{v}^{u} k_{i, j}(x, s, \mathbf{f}(s)) d s(i, j=1,2, \ldots, n)$ and define

$$
\begin{aligned}
T_{i, j}^{(0)}(u, v) & :=\frac{u-v}{2}\left[k_{i, j}\left(x, v, \mathbf{F}_{v}\right)+k_{i, j}\left(x, u, \mathbf{F}_{u}\right)\right], \\
T_{i, j}^{(1)}(u, v) & :=\frac{1}{2} T_{i, j}^{(0)}(u, v)+\frac{u-v}{2} k_{i, j}\left(x, \frac{u+v}{2}, \mathbf{F}_{\frac{u+v}{2}}\right), \\
T_{i, j}^{(2)}(u, v) & :=\frac{1}{2} T_{i, j}^{(1)}(u, v)+\frac{u-v}{4}\left[k_{i, j}\left(x, \frac{u+3 v}{4}, \mathbf{F}_{\frac{u+3 v}{4}}\right)+k_{i, j}\left(x, \frac{3 u+v}{4}, \mathbf{F}_{\frac{3 u+v}{4}}\right)\right], \\
T_{i, j}^{(3)}(u, v) & :=\frac{1}{2} T_{i, j}^{(2)}(u, v)+\frac{u-v}{8}\left[k_{i, j}\left(x, \frac{u+7 v}{8}, \mathbf{F}_{\frac{u+7 v}{8}}\right)+k_{i, j}\left(x, \frac{3 u+5 v}{8}, \mathbf{F}_{\frac{3 u+5 v}{8}}\right)\right. \\
& \left.+k_{i, j}\left(x, \frac{5 u+3 v}{8}, \mathbf{F}_{\frac{5 u+3 v}{8}}\right)+k_{i, j}\left(x, \frac{7 u+v}{8}, \mathbf{F}_{\frac{7 u+v}{8}}\right)\right],
\end{aligned}
$$

where $T_{i, j}^{(l)}(u, v)(l=0,1,2,3)$, denotes the trapezoidal rule with $2^{l}$ subintervals and $\mathbf{F}_{\mu}=\left(F_{1, \mu}, F_{2, \mu}, \ldots, F_{n, \mu}\right) \approx\left(f_{1}\left(x_{\mu}\right), f_{2}\left(x_{\mu}\right), \ldots, f_{n}\left(x_{\mu}\right)\right)$ for $\mu=$ $v, \frac{u+7 v}{8}, \ldots, u$. Therefore two and three stages Romberg quadrature rules can be respectively written as

$$
\int_{v}^{u} k_{i, j}(x, s, \mathbf{f}(s)) d s \approx \frac{64}{45} T_{i, j}^{(2)}(u, v)-\frac{20}{45} T_{i, j}^{(1)}(u, v)+\frac{1}{45} T_{i, j}^{(0)}(u, v)=: A_{i, j}(u, v)
$$


and

$$
\begin{aligned}
\int_{v}^{u} k_{i, j}(x, s, \mathbf{f}(s)) d s \approx & \frac{4096}{2835} T_{i, j}^{(3)}(u, v)-\frac{1344}{2835} T_{i, j}^{(2)}(u, v)+\frac{84}{2835} T_{i, j}^{(1)}(u, v) \\
& -\frac{1}{2835} T_{i, j}^{(0)}(u, v)=: B_{i, j}(u, v) .
\end{aligned}
$$

\subsection{Description of the method}

Let $x_{m, l}=m h+u_{l} h, l=0,1, \ldots, p, m=0,1, \ldots, N-1$ with $N, p \in \mathbb{N}, h>$ $0, N h=X$ and $0 \leq u_{0}<u_{1}<\ldots<u_{p} \leq 1$. Then put $x=x_{m, l}$ in eq. (2.1) and write it in the form

$$
\begin{aligned}
\mathbf{f}\left(x_{m, l}\right)=\mathbf{g}\left(x_{m, l}\right)+\int_{0}^{x_{m, 0}} \mathbf{K}\left(x_{m, l}, s, \mathbf{f}(s)\right) \mathbf{H}\left(x_{m, l}, s\right) d s \\
+\int_{x_{m, 0}}^{x_{m, l}} \mathbf{K}\left(x_{m, l}, s, \mathbf{f}(s)\right) \mathbf{H}\left(x_{m, l}, s\right) d s .
\end{aligned}
$$

Let $F_{i, m, l} \simeq f_{i}\left(x_{m, l}\right)$ for $i=0,1, \ldots, n$. If $\mathbf{F}_{0, l}, \ldots, \mathbf{F}_{m-1, l} \in \mathbb{R}^{n}(l=0,1, \ldots, p)$ with $\mathbf{F}_{j, l}=\left[F_{1, j, l}, F_{2, j, l}, \ldots, F_{n, j, l}\right]^{T}(j=0,1, \ldots, m-1)$ are known, then the first integral in equation (2.5) can be approximated by standard quadrature rules and the second one is estimated by Romberg quadrature rule. Thus a system of equations will be obtained. In what follows, we describe a method with four blocks which has at least six order of convergence. This can be generalized by increasing the number of blocks to obtain a method with higher order of convergence. Thus let $p=4$ and $u_{l}=l / 4$ for $l=0,1, \ldots, 4$.

At first, we describe the method for a single VIE, then we generalize it to the system of VIEs. Thus, we put $n=1$ in eq. (2.5) and obtain

$$
\begin{gathered}
f_{1}\left(x_{0,0}\right)=g\left(x_{0,0}\right), \quad f_{1}\left(x_{m, l}\right)=g_{1}\left(x_{m, l}\right)+\int_{0}^{x_{m, 0}} k_{1,1}\left(x_{m, l}, s, f_{1}(s)\right) d s \\
\quad+\int_{x_{m, 0}}^{x_{m, l}} k_{1,1}\left(x_{m, l}, s, f_{1}(s)\right) d s, \quad m=0,1, \ldots, N-1, l=1,2,3,4 .
\end{gathered}
$$

Then we use eq. (2.3) for the second integral and obtain

$$
\begin{aligned}
& \int_{x_{m, 0}}^{x_{m, l}} k_{1,1}\left(x_{m, l}, s, f_{1}(s)\right) d s \approx A_{1,1}\left(\left(m+\frac{l}{4}\right) h, m h\right)=\frac{l h / 4}{90}\left[7 \left[k_{1,1}\left(x_{m, l}, x_{m, 0}, F_{1, m, 0}\right)\right.\right. \\
& \left.\quad+k_{1,1}\left(x_{m, l}, x_{m, l}, F_{1, m, l}\right)\right]+12 k_{1,1}\left(x_{m, l}, x_{m, \frac{l}{2}}, F_{1, m, \frac{l}{2}}\right) \\
& \quad+32\left[k_{1,1}\left(x_{m, l}, x_{m, \frac{l}{4}}, F_{1, m, \frac{l}{4}}\right)+k_{1,1}\left(x_{m, l}, x_{m, \frac{3 l}{4}}, F_{1, m, \frac{3 l}{4}}\right]\right], l=1,2,3,4 . \quad \text { (2.7) }
\end{aligned}
$$

If $\frac{j l}{4} \notin\left\{0, \frac{1}{4}, \frac{1}{2}, \frac{3}{4}, 1\right\}$ for some $j \in\{1,2,3\}$, then $F_{1, m, \frac{j l}{4}}$ make a difficulty in computing $f_{1}\left(x_{m, l}\right)$. For such a case, we use the Lagrange interpolation at the points $x_{m, 0}, x_{m, 1}, x_{m, 2}, x_{m, 3}, x_{m, 4}$ and obtain

$$
F_{i, m, j l / 4} \approx \mathcal{P}_{i}\left(x_{m}, \frac{j l}{4}\right)=\sum_{q=0}^{4} L_{q}(j l / 4) F_{i, m, q}, \quad L_{q}(x)=\prod_{\substack{t=0 \\ t \neq q}}^{4} \frac{x-t}{q-t}, \quad j=1,2,3,
$$


where $L_{l}(x)$ is the fundamental Lagrange polynomial. When $x$ changes in a large interval or the step size is very small, we need more accurate approximation for the first integral in eq. (2.5) to reduce the effect of accumulated errors in evaluating the unknown function at the points near to the end of interval. Therefore, it is better the three-stage Romberg rule, $B_{i, j}(u, v)$, be used to approximate this integral. To do this, we consider the following cases.

Case(I): If $m$ is even, then

$$
\begin{aligned}
\int_{0}^{x_{m, 0}} & k_{1,1}\left(x_{m, l}, s, f_{1}(s)\right) d s \approx B_{1,1}(m h, 0) \\
& =\frac{x_{m, 0}}{2835}\left[108.5\left[k_{1,1}\left(x_{m, l}, 0, F_{1,0,0}\right)+k_{1,1}\left(x_{m, l}, x_{m, 0}, F_{1, m, 0}\right)\right]\right. \\
& +218 k_{1,1}\left(x_{m, l}, x_{m / 2,0}, F_{1, m / 2,0}\right) \\
& +176\left[k_{1,1}\left(x_{m, l}, x_{m / 4,0}, F_{1, m / 4,0}\right)+k_{1,1}\left(x_{m, l}, x_{3 m / 4,0}, F_{1,3 m / 4,0}\right)\right] \\
& +512\left[k_{1,1}\left(x_{m, l}, x_{m / 8,0}, F_{1, m / 8,0}\right)+k_{1,1}\left(x_{m, l}, x_{3 m / 8,0}, F_{1,3 m / 8,0}\right)\right. \\
& \left.\left.+k_{1,1}\left(x_{m, l}, x_{5 m / 8,0}, F_{1,5 m / 8,0}\right)+k_{1,1}\left(x_{m, l}, x_{7 m / 8,0}, F_{1,7 m / 8,0}\right)\right]\right]
\end{aligned}
$$

and substituting it in eq. (2.6) yields

$$
F_{1, m, l}=g_{1}\left(x_{m, l}\right)+B_{1,1}(m h, 0)+A_{1,1}((m+l / 4) h, m h), \quad l=1,2,3,4 .
$$

Case(II): If $m$ is odd, then

$$
\begin{aligned}
\int_{0}^{x_{m, 0}} & k_{1,1}\left(x_{m, l}, s, f_{1}(s)\right) d s \\
& =\int_{0}^{x_{1,0}} k_{1,1}\left(x_{m, l}, s, f_{1}(s)\right) d s+\int_{x_{1,0}}^{x_{m, 0}} k_{1,1}\left(x_{m, l}, s, f_{1}(s)\right) d s \\
& \approx A_{1,1}(h, 0)+B_{1,1}(m h, h)=\frac{x_{1}}{90}\left[12 k_{1,1}\left(x_{m, l}, x_{\frac{1}{2}, 0}, F_{1, \frac{1}{2}, 0}\right)\right. \\
& +7\left[k_{1,1}\left(x_{m, l}, x_{1,0}, F_{1,1,0}\right)+k_{1,1}\left(x_{m, l}, 0, F_{1,0,0}\right)\right] \\
& \left.+32\left[k_{1,1}\left(x_{m, l}, x_{\frac{1}{4}, 0}, F_{1, \frac{1}{4}, 0}\right)+k_{1,1}\left(x_{m, l}, x_{\frac{3}{4}, 0}, F_{1, \frac{3}{4}, 0}\right)\right]\right] \\
& +\frac{(m-1) h}{2835}\left[108.5\left[k_{1,1}\left(x_{m, l}, x_{1,0}, F_{1,1,0}\right)+k_{1,1}\left(x_{m, l}, x_{m, 0}, F_{1, m, 0}\right)\right]\right. \\
& +218 k_{1,1}\left(x_{m, l}, x_{(1+m) / 2,0}, F_{1,(1+m) / 2,0}\right) \\
& +176\left[k_{1,1}\left(x_{m, l}, x_{\frac{3+m}{4}, 0}, F_{1, \frac{3+m}{4}, 0}\right)+k_{1,1}\left(x_{m, l}, x_{\frac{1+3 m}{4}, 0}, F_{1, \frac{1+3 m}{4}, 0}\right)\right] \\
& +512\left[k_{1,1}\left(x_{m, l}, x_{\frac{7+m}{8}, 0}, F_{1, \frac{7+m}{8}, 0}\right)+k_{1,1}\left(x_{m, l}, x_{\frac{5+3 m}{8}, 0}, F_{1, \frac{5+3 m}{8}, 0}\right)\right. \\
& \left.\left.+k_{1,1}\left(x_{m, l}, x_{\frac{3+5 m}{8}, 0}, F_{1, \frac{3+5 m}{8}, 0}\right)+k_{1,1}\left(x_{m, l}, x_{\frac{1+7 m}{8}, 0}, F_{1, \frac{1+7 m}{8}, 0}\right)\right]\right]
\end{aligned}
$$

and substituting this again in (2.6) yields

$$
\begin{array}{r}
F_{1, m, l}=g_{1}\left(x_{m, l}\right)+A_{1,1}(h, 0)+B_{1,1}(m h, h)+A_{1,1}((m+l / 4) h, m h), \\
l=1,2,3,4 .
\end{array}
$$

Therefore, in each case a system of 4 equations is obtained which is solved for the unknowns $F_{1, m, 1}, F_{1, m, 2}, F_{1, m, 3}$ and $F_{1, m, 4}$. 


\subsection{The general process}

Now, let us assume that $\mathbf{f}, \mathbf{g}$ and $\mathbf{H}$ are vectors with $n$ component (where $\left.H_{j}(t, s)=1, j=1,2, \ldots, n\right)$ and $\mathbf{K}$ is an $n \times n$ matrix and the grid points are defined similar to the case $n=1$. Then eq.(2.5) can be written as

$$
\begin{aligned}
& f_{1}\left(x_{m, l}\right)=g_{1}\left(x_{m, l}\right)+\int_{0}^{x_{m, 0}} \sum_{j=1}^{n} k_{1, j}\left(x_{m, l}, s, \mathbf{f}(s)\right) d s \\
&+\int_{x_{m, 0}}^{x_{m, l}} \sum_{j=1}^{n} k_{1, j}\left(x_{m, l}, s, \mathbf{f}(s)\right) d s, \\
& f_{2}\left(x_{m, l}\right)=g_{2}\left(x_{m, l}\right)+\int_{0}^{x_{m, 0}} \sum_{j=1}^{n} k_{2, j}\left(x_{m, l}, s, \mathbf{f}(s)\right) d s+\int_{x_{m, 0}}^{x_{m, l}} \sum_{j=1}^{n} k_{2, j}\left(x_{m, l}, s, \mathbf{f}(s)\right) d s, \\
& \ldots \\
& f_{n}\left(x_{m, l}\right)=g_{n}\left(x_{m, l}\right)+\int_{0}^{x_{m, 0}} \sum_{j=1}^{n} k_{n, j}\left(x_{m, l}, s, \mathbf{f}(s)\right) d s \\
&+\int_{x_{m, 0}}^{x_{m, l}} \sum_{j=1}^{n} k_{n, j}\left(x_{m, l}, s, \mathbf{f}(s)\right) d s .
\end{aligned}
$$

To approximate the integrals in eq. (2.10), we use (2.3) and (2.4) from previous subsection. Therefore for the even values of $m$, we obtain

$$
\begin{aligned}
\mathbf{F}_{0,0} & =\mathbf{g}\left(x_{0,0}\right), \\
F_{1, m, l} & =g_{1}\left(x_{m, l}\right)+\sum_{j=0}^{n}\left[B_{1, j}(m h, 0)+A_{1, j}((m+l / 4) h, m h)\right], \\
F_{2, m, l} & =g_{2}\left(x_{m, l}\right)+\sum_{j=0}^{n}\left[B_{2, j}(m h, 0)+A_{2, j}((m+l / 4) h, m h)\right], \\
\ldots & \\
F_{n, m, l} & =g_{n}\left(x_{m, l}\right)+\sum_{j=0}^{n}\left[B_{n, j}(m h, 0)+A_{n, j}((m+l / 4) h, m h)\right], \\
& \quad m=0,1, \ldots, N-1, l=1,2,3,4,
\end{aligned}
$$

otherwise (when $m$ is odd), we obtain

$$
\begin{aligned}
& F_{1, m, l}=g_{1}\left(x_{m, l}\right)+\sum_{j=1}^{n}\left[A_{1, j}(h, 0)+A_{1, j}((m+l / 4) h, m h)+B_{1, j}(m h, h)\right], \\
& F_{2, m, l}=g_{2}\left(x_{m, l}\right)+\sum_{j=1}^{n}\left[A_{2, j}(h, 0)+A_{2, j}((m+l / 4) h, m h)+B_{2, j}(m h, h)\right],
\end{aligned}
$$




$$
F_{n, m, l}=g_{n}\left(x_{m, l}\right)+\sum_{j=1}^{n}\left[A_{n, j}(h, 0)+A_{n, j}((m+l / 4) h, m h)+B_{n, j}(m h, h)\right],
$$

or equivalently

$$
\begin{aligned}
& {\left[\begin{array}{l}
F_{1, m, l}-\sum_{j=1}^{n} A_{1, j}((m+l / 4) h, m h) \\
F_{2, m, l}-\sum_{j=1}^{n} A_{2, j}((m+l / 4) h, m h) \\
\cdots \\
F_{n, m, l}-\sum_{j=1}^{n} A_{n, j}((m+l / 4) h, m h)
\end{array}\right]=} \\
& {\left[\begin{array}{c}
g_{1}\left(x_{m, l}\right)+\sum_{j=1}^{n}\left[A_{1, j}(h, 0)+B_{1, j}(m h, h)\right] \\
g_{2}\left(x_{m, l}\right)+\sum_{j=1}^{n}\left[A_{2, j}(h, 0)+B_{2, j}(m h, h)\right] \\
\cdots \\
g_{n}\left(x_{m, l}\right)+\sum_{j=1}^{n}\left[A_{n, j}(h, 0)+B_{n, j}(m h, h)\right]
\end{array}\right]}
\end{aligned}
$$

for $m=0,1,2, \ldots, N-1$ and $l=1, \ldots, 4$. Consequently, at each step, we get a system of $4 n$ equations with the unknowns $F_{1, m, 1}, F_{1, m, 2}, \ldots, F_{1, m, 4}, F_{2, m, 1}$, $\ldots, F_{2, m, 4}, \ldots, F_{n, m, 1}, \ldots, F_{n, m, 4}$, which will be linear and nonlinear respectively for linear and nonlinear integral equations. For the linear case it is solved via a direct method but for the nonlinear case, the system may be solved by using an iterative method or by using a suitable software package such as Maple.

\subsection{Convergence analysis}

Theorem 1. The approximation method given by the system (2.11) is convergent and its order of convergence is at least 6 for the functions $k_{i, j}$ and $f_{i}$ $(i, j=1,2, \ldots, n)$ with at least six-order continuous derivations.

Proof. Define $\mathbf{e}_{m, l}:=\mathbf{F}_{m, l}-\mathbf{f}\left(x_{m, l}\right)$, where $\mathbf{f}\left(x_{m, l}\right) \in \mathbb{R}^{n}$ and $\mathbf{F}_{m, l} \in \mathbb{R}^{n}$ denote respectively the exact and approximate solutions of (1.1) at the point $x=x_{m, l}$. From (2.8) and (2.9) we have

$$
\int_{0}^{x_{m, 0}} \mathbf{K}\left(x_{m, l}, s, \mathbf{f}(s)\right) d s \approx h \sum_{i=0}^{m-1} \sum_{j=0}^{4} w_{i j} \mathbf{K}\left(x_{m, l}, x_{i, j}, \mathbf{F}_{i, j}\right) .
$$

Then subtracting (2.5) from (2.7) and (2.12) (for $l=1$ ) gives

$$
\begin{aligned}
\left\|\mathbf{e}_{m, 1}\right\|_{1} & =\left\|\mathbf{F}_{m, 1}-\mathbf{f}\left(x_{m, 1}\right)\right\|_{1}=\| h \sum_{i=0}^{m-1} \sum_{j=0}^{4} w_{i j} \mathbf{K}\left(x_{m, 1}, x_{i, j}, \mathbf{F}_{i, j}\right) \\
& +\frac{7}{90} x_{0,1}\left[\mathbf{K}\left(x_{m, 1}, x_{m, 0}, \mathbf{F}_{m, 0}\right)+\mathbf{K}\left(x_{m, 1}, x_{m, l}, \mathbf{F}_{m, 1}\right)\right] \\
& +\frac{6}{45} x_{0,1} \mathbf{K}\left(x_{m, 1}, x_{m, 1 / 2}, \mathcal{P}\left(x_{m}, 1 / 2\right)\right) \\
& +\frac{16}{45} x_{0,1}\left[\mathbf{K}\left(x_{m, 1}, x_{m, 1 / 4}, \mathcal{P}\left(x_{m}, 1 / 4\right)\right)+\mathbf{K}\left(x_{m, 1}, x_{m, \frac{3}{4}}, \mathcal{P}\left(x_{m}, \frac{3}{4}\right)\right)\right] \\
& -\int_{0}^{x_{m, 0}} \mathbf{K}\left(x_{m, 1}, s, \mathbf{f}(s)\right) d s-\int_{x_{m, 0}}^{x_{m, 1}} \mathbf{K}\left(x_{m, 1}, s, \mathbf{f}(s)\right) d s \|_{1}, m=0,1, \ldots, N-1,
\end{aligned}
$$


where $\mathcal{P}(x, s)=\left[\mathcal{P}_{1}(x, s), \ldots, \mathcal{P}_{n}(x, s)\right]$. By adding and diminishing the terms

$$
\sum_{i=0}^{m-1} \sum_{j=0}^{4} w_{i j} \mathbf{K}\left(x_{m, 1}, x_{i, j}, \mathbf{f}\left(x_{i, j}\right)\right), \ldots, \frac{16}{45} x_{0,1} \mathbf{K}\left(x_{m, 1}, x_{m, \frac{3}{4}}, \sum_{q=0}^{4} L_{q}\left(\frac{3}{4}\right) \mathbf{f}\left(x_{m, q}\right)\right)
$$

and using the conditions of Theorem 1, one can write

$$
\begin{aligned}
\left\|\mathbf{e}_{m, 1}\right\|_{1} \leq h \sum_{i=0}^{m-1} \sum_{j=0}^{4} w_{i, j} Q\left(x_{m, 1}, x_{i, j}\right)\left\|\mathbf{f}\left(x_{i, j}\right)-\mathbf{F}_{i, j}\right\|_{1}+\frac{7}{90} x_{0,1} Q\left(x_{m, 1}, x_{m, 0}\right) \\
\quad \times\left\|\mathbf{f}\left(x_{m, 0}\right)-\mathbf{F}_{m, 0}\right\|_{1}+\frac{7}{90} x_{0,1} Q\left(x_{m, 1}, x_{m, 1}\right)\left\|\mathbf{f}\left(x_{m, 1}\right)-\mathbf{F}_{m, 1}\right\|_{1} \\
\quad+\frac{6}{45} x_{0,1} Q\left(x_{m, 1}, x_{m, \frac{1}{2}}\right)\left\|\sum_{q=0}^{4} L_{q}\left(\frac{1}{2}\right)\left(\mathbf{f}\left(x_{m, q}\right)-\mathbf{F}_{m, q}\right)\right\|_{1} \\
\quad+\frac{16}{45} x_{0,1} Q\left(x_{m, 1}, x_{m, \frac{1}{4}}\right)\left\|\sum_{q=0}^{4} L_{q}\left(\frac{1}{4}\right)\left(\mathbf{f}\left(x_{m, q}\right)-\mathbf{F}_{m, q}\right)\right\|_{1} \\
\quad+\frac{16}{45} x_{0,1} Q\left(x_{m, 1}, x_{m, \frac{3}{4}}\right)\left\|\sum_{q=0}^{4} L_{q}\left(\frac{3}{4}\right)\left(\mathbf{f}\left(x_{m, q}\right)-\mathbf{F}_{m, q}\right)\right\|_{1}+\left\|R_{1}\right\|_{1}+\left\|R_{2}\right\|_{1},
\end{aligned}
$$

where $R_{1}$ and $R_{2}$ are the errors of numerical integrations. Since $Q(t, s)$ is continuous, one can write $\left|Q\left(x_{m, 1}, x_{i, j}\right)\right| \leq C_{i j}$, thus

$$
\begin{aligned}
\left\|\mathbf{e}_{m, 1}\right\|_{1} & \leq h \sum_{i=0}^{m-1} \sum_{j=0}^{4} w_{i, j} C_{i j}\left\|\mathbf{e}_{i, j}\right\|_{1}+h c_{0}\left\|\mathbf{e}_{m, 0}\right\|_{1}+h c_{1}\left\|\mathbf{e}_{m, 1}\right\|_{1}+h c_{2}\left\|\mathbf{e}_{m, 2}\right\|_{1} \\
& +h c_{3}\left\|\mathbf{e}_{m, 3}\right\|_{1}+h c_{4}\left\|\mathbf{e}_{m, 4}\right\|_{1}+\left\|R_{1}\right\|_{1}+\left\|R_{2}\right\|_{1},
\end{aligned}
$$

where $c_{i}(i=0,1, \ldots, 4)$ are constants. For other values of $l$, similarly we have

$$
\begin{aligned}
\left\|\mathbf{e}_{m, l}\right\|_{1} & \leq h \sum_{i=0}^{m-1} \sum_{j=0}^{4} w_{i, j} C_{i j}\left\|\mathbf{e}_{i, j}\right\|_{1}+h c_{0}\left\|\mathbf{e}_{m, 0}\right\|_{1}+h c_{1}\left\|\mathbf{e}_{m, 1}\right\|_{1}+h c_{2}\left\|\mathbf{e}_{m, 2}\right\|_{1} \\
& +h c_{3}\left\|\mathbf{e}_{m, 3}\right\|_{1}+h c_{4}\left\|\mathbf{e}_{m, 4}\right\|_{1}+\left\|R_{1}\right\|_{1}+\left\|R_{2}\right\|_{1}, \quad l=2,3,4 .
\end{aligned}
$$

Let $\left\|\mathbf{e}_{m, 1}\right\|_{1}=\max _{l=1,2,3,4}\left\|\mathbf{e}_{m, l}\right\|_{1}$. Therefore

$$
\left\|\mathbf{e}_{m, 1}\right\|_{1} \leq c h \sum_{i=0}^{m-1}\left\|\mathbf{e}_{i, 1}\right\|_{1}+h c^{\prime}\left\|\mathbf{e}_{m, 1}\right\|_{1}+\left\|R_{1}\right\|_{1}+\left\|R_{2}\right\|_{1},
$$

where $c^{\prime}=c_{1}+c_{2}+c_{3}+c_{4}$ and $c$ are constants. Then for sufficiently small $h$

$$
\left\|\mathbf{e}_{m, 1}\right\|_{1} \leq \frac{h c}{1-h c^{\prime}} \sum_{i=0}^{m-1}\left\|\mathbf{e}_{i, 1}\right\|_{1}+\frac{\left\|R_{1}\right\|_{1}+\left\|R_{2}\right\|_{1}}{1-h c^{\prime}}
$$

and using the Gronwall inequality (see [14], for example), yields

$$
\left\|\mathbf{e}_{m, 1}\right\|_{1} \leq \frac{\left\|R_{1}\right\|_{1}+\left\|R_{2}\right\|_{1}}{1-h c^{\prime}} e^{\frac{c}{1-h c^{\prime}} x_{m}},
$$


hence $\left\|\mathbf{e}_{m, 1}\right\|_{1} \rightarrow 0$ as $h \rightarrow 0$ since $R_{i}(i=1,2)$ tends to zero as $h \rightarrow 0$. For the functions $k_{i, j}$ and $f_{i}(i, j=1,2, \ldots, n)$ with at least 6 order continuous derivatives, we have $\left\|R_{1}\right\|_{1},\left\|R_{2}\right\|_{1}=O\left(h^{6}\right)$ and so $\left\|\mathbf{e}_{m, 1}\right\|_{1}=O\left(h^{6}\right)$ which completes the proof.

\section{System of Abel VIEs}

Consider a system of nonlinear Volterra integral equations of the Abel types, i.e.

$$
\mathbf{f}(x)=\mathbf{g}(x)+\int_{0}^{x} \mathbf{K}(x, s, \mathbf{f}(s)) \mathbf{H}(x, s) d s, \quad 0 \leq s \leq x \leq X
$$

with $H_{j}(x, s)=\frac{1}{|x-s|^{\alpha_{j}}}, 0 \leq \alpha_{j}<1, j=1,2, \ldots, n\left(\alpha_{j}=0\right.$ described in the previous section).

\subsection{Existence and uniqueness of solution}

Theorem 2. Let:

(i) $\mathbf{g}(x)$ is continuous (i.e. every component is continuous),

(ii) $\mathbf{K}(x, s, \mathbf{f}(s))$ satisfies Lipshitz condition, i.e. for fixed $x, s$ with $0 \leq s \leq$ $x \leq X$ there is a positive constant $l$ independent of $s$ and $x$, such that

$$
\|\mathbf{K}(x, s, \mathbf{y})-\mathbf{K}(x, s, \mathbf{z})\| \leq l\|\mathbf{y}-\mathbf{z}\| \quad \forall \mathbf{y}, \mathbf{z} \in \mathbb{R}^{n},
$$

then (3.1) has a unique solution.

Proof. See [17].

\subsection{Description of the method}

Similar to the previous section, define $x_{m, l}=m h+u_{l} h, l=0,1, \ldots, p ; m=$ $0,1, \ldots, N-1,(N, p \in \mathbb{N}, h>0$,$) so that N h=X$ and $0 \leq u_{0}<u_{1}<\ldots<u_{p}$. Substituting $x=x_{m, l}$ in (3.1), we have

$$
\begin{aligned}
\mathbf{f}\left(x_{m, l}\right) & =\mathbf{g}\left(x_{m, l}\right)+\int_{0}^{x_{m, l}} \mathbf{K}\left(x_{m, l}, s, \mathbf{f}(s)\right) \mathbf{H}\left(x_{m, l}, s\right) d s \\
& =\mathbf{g}\left(x_{m, l}\right)+\int_{0}^{x_{m, 0}} \mathbf{K}\left(x_{m, l}, s, \mathbf{f}(s)\right) \mathbf{H}\left(x_{m, l}, s\right) d s \\
& +\int_{x_{m, 0}}^{x_{m, l}} \mathbf{K}\left(x_{m, l}, s, \mathbf{f}(s)\right) \mathbf{H}\left(x_{m, l}, s\right) d s .
\end{aligned}
$$

The method of the previous section can't be used for this system, because of the singularity of $K_{i, j}\left(x_{m, l}, s, \mathbf{f}(s)\right) H_{j}\left(x_{m, l}, s\right)$ at $s=x_{m, l}$. So we use the following product integration method instead of a usual integration method. We write

$$
f_{i}\left(x_{m, l}\right)=g_{i}\left(x_{m, l}\right)+\int_{0}^{x_{m, 0}} \sum_{j=1}^{n} K_{i, j}\left(x_{m, l}, s, \mathbf{f}(s)\right) H_{j}\left(x_{m, l}, s\right) d s
$$




$$
+\int_{x_{m, 0}}^{x_{m, l}} \sum_{j=1}^{n} K_{i, j}\left(x_{m, l}, s, \mathbf{f}(s)\right) H_{j}\left(x_{m, l}, s\right) d s, \quad i=1,2, \ldots, n
$$

and use the Lagrange interpolation, to write

$$
\begin{aligned}
& K_{i, j}\left(x_{m, l}, s, \mathbf{f}(s)\right) \approx \sum_{t=0}^{p} L_{t}(s) K_{i, j}\left(x_{m, l}, x_{m, t}, \mathbf{f}\left(x_{m, t}\right)\right), \\
& L_{t}(s)=\prod_{q=0, q \neq t}^{p} \frac{s-x_{m, q}}{x_{m, t}-x_{m, q}}, \quad t=0,1, \cdots, p
\end{aligned}
$$

then

$$
\begin{aligned}
& \int_{x_{m, 0}}^{x_{m, l}}\left(K_{i, j}\left(x_{m, l}, s, \mathbf{f}(s)\right) H_{j}\left(x_{m, l}, s\right)\right) d s \\
& \quad \approx \sum_{t=0}^{p} K_{i, j}\left(x_{m, l}, x_{m, t}, \mathbf{f}\left(x_{m, t}\right)\right) \frac{\int_{x_{m, 0}}^{x_{m, l}}\left(\prod_{q=0, q \neq t}^{p}\left(s-x_{m, q}\right) H_{j}\left(x_{m, l}, s\right)\right) d s}{\prod_{q=0, q \neq t}^{p}\left(x_{m, t}-x_{m, q}\right)}
\end{aligned}
$$

By using the variable transformation $s=x_{m}+l S h / p$, one can write

$$
\begin{aligned}
\int_{x_{m, 0}}^{x_{m}, l} & \prod_{q=0, q \neq t}^{p}\left(s-x_{m, q}\right) H_{j}\left(x_{m, l}, s\right) d s \\
& =l\left(\frac{h}{p}\right)^{p+1} \int_{0}^{1} \prod_{q=0, q \neq t}^{p}(l S-q) H_{j}\left(x_{m, l}, x_{m}+\frac{l h S}{p}\right) d S, \quad l=1,2, \ldots, p .
\end{aligned}
$$

For simplicity, we set $p=4$ and $u_{l}=l / 4,(l=1,2, \ldots, 4)$, and obtain from $(3.3)$

$$
\begin{aligned}
L_{0}(s) & =\frac{32}{3 h^{4}}\left(s-x_{m, 1}\right)\left(s-x_{m, 2}\right)\left(s-x_{m, 3}\right)\left(s-x_{m, 4}\right), \\
L_{1}(s) & =\frac{-128}{3 h^{4}}\left(s-x_{m, 0}\right)\left(s-x_{m, 2}\right)\left(s-x_{m, 3}\right)\left(s-x_{m, 4}\right), \\
& \cdots \\
L_{4}(s) & =\frac{32}{3 h^{4}}\left(s-x_{m, 0}\right)\left(s-x_{m, 1}\right)\left(s-x_{m, 2}\right)\left(s-x_{m, 3}\right),
\end{aligned}
$$

then by using the variable transformation $s=\left(m+\frac{l S}{4}\right) h$, we write (3.4) as

$$
\begin{aligned}
& \int_{x_{m, 0}}^{x_{m, l}} K_{i, j}\left(x_{m, l}, s, \mathbf{f}(s)\right) H_{j}\left(x_{m, l}, s\right) d s \approx K_{i, j}\left(x_{m, l}, x_{m, 0}, \mathbf{F}_{m, 0}\right) \zeta_{j}\left(x_{m, l}, x_{m}, l h / 4\right) \\
& \quad+K_{i, j}\left(x_{m, l}, x_{m, 1}, \mathbf{F}_{m, 1}\right) \beta_{j}\left(x_{m, l}, x_{m}, l h / 4\right)+K_{i, j}\left(x_{m, l}, x_{m, 2}, \mathbf{F}_{m, 2}\right) \\
& \quad \times \gamma_{j}\left(x_{m, l}, x_{m}, l h / 4\right)+K_{i, j}\left(x_{m, l}, x_{m, 3}, \mathbf{F}_{m, 3}\right) \theta_{j}\left(x_{m, l}, x_{m}, l h / 4\right) \\
& \quad+K_{i, j}\left(x_{m, l}, x_{m, 4}, \mathbf{F}_{m, 4}\right) \eta_{j}\left(x_{m, l}, x_{m}, l h / 4\right)
\end{aligned}
$$


where

$$
\begin{aligned}
\zeta_{j}(x, y, z) & :=\frac{z}{24} \int_{0}^{1}\left[(l S-1)(l S-2)(l S-3)(l S-4) H_{j}(x, y+S z)\right] d S, \\
\beta_{j}(x, y, z) & :=\frac{-z}{6} \int_{0}^{1}\left[l S(l S-2)(l S-3)(l S-4) H_{j}(x, y+S z)\right] d S, \\
\gamma_{j}(x, y, z) & :=\frac{z}{4} \int_{0}^{1}\left[l S(l S-1)(l S-3)(l S-4) H_{j}(x, y+S z)\right] d S \\
\theta_{j}(x, y, z) & :=\frac{-z}{6} \int_{0}^{1}\left[l S(l S-1)(l S-2)(l S-4) H_{j}(x, y+S z)\right] d S, \\
\eta_{j}(x, y, z) & :=\frac{z}{24} \int_{0}^{1}\left[l S(l S-1)(l S-2)(l S-3) H_{j}(x, y+S z)\right] d S, \quad l=1, \ldots, 4 .
\end{aligned}
$$

Thus similar to the previous section, we distinguish the following cases in (3.2).

Case(I): When $m$ is even, we use (2.4) to get

$$
\begin{gathered}
\int_{0}^{x_{m, 0}} \quad k_{i, j}\left(x_{m, l}, s, \mathbf{f}(s)\right) H_{j}\left(x_{m, l}, s\right) d s \approx \frac{x_{m, 0}}{2835}\left[1 0 8 . 5 \left[\frac{k_{i, j}\left(x_{m, l}, 0, \mathbf{F}_{0,0}\right)}{\left|x_{m, l}-0\right|^{\alpha_{j}}}\right.\right. \\
\left.+\frac{k_{i, j}\left(x_{m, l}, x_{m, 0}, \mathbf{F}_{m, 0}\right)}{\left|x_{m, l}-x_{m, 0}\right|^{\alpha_{j}}}\right]+218 \frac{k_{i, j}\left(x_{m, l}, x_{m / 2,0}, \mathbf{F}_{m / 2,0}\right)}{\left|x_{m, l}-x_{m / 2,0}\right|^{\alpha_{j}}} \\
+176\left[\frac{k_{i, j}\left(x_{m, l}, x_{m / 4,0}, \mathbf{F}_{m / 4,0}\right)}{\left|x_{m, l}-x_{m / 4,0}\right|^{\alpha_{j}}}+\frac{k_{i, j}\left(x_{m, l}, x_{3 m / 4,0}, \mathbf{F}_{3 m / 4,0}\right)}{\left|x_{m, l}-x_{3 m / 4,0}\right|^{\alpha_{j}}}\right] \\
+512\left[\frac{k_{i, j}\left(x_{m, l}, x_{m / 8,0}, \mathbf{F}_{m / 8,0}\right)}{\left|x_{m, l}-x_{m / 8,0}\right|^{\alpha_{j}}}+\frac{k_{i, j}\left(x_{m, l}, x_{3 m / 8,0}, \mathbf{F}_{3 m / 8,0}\right)}{\left|x_{m, l}-x_{3 m / 8,0}\right|^{\alpha_{j}}}\right. \\
\left.\left.+\frac{k_{i, j}\left(x_{m, l}, x_{5 m / 8,0}, \mathbf{F}_{5 m / 8,0}\right)}{\left|x_{m, l}-x_{5 m / 8,0}\right|^{\alpha_{j}}}+\frac{k_{i, j}\left(x_{m, l}, x_{7 m / 8,0}, \mathbf{F}_{7 m / 8,0}\right)}{\left|x_{m, l}-x_{7 m / 8,0}\right|^{\alpha_{j}}}\right]\right] .
\end{gathered}
$$

Case(II): When $m$ is odd, we use (2.3) and (2.4) to get

$$
\begin{aligned}
& \int_{0}^{x_{m, 0}} \frac{k_{i, j}\left(x_{m, l}, s, \mathbf{f}(s)\right)}{\left|x_{m, l}-s\right|^{\alpha_{j}}} d s=\int_{0}^{x_{1,0}} \frac{k_{i, j}\left(x_{m, l}, s, \mathbf{f}(s)\right)}{\left|x_{m, l}-s\right|^{\alpha_{j}}} d s+\int_{x_{1,0}}^{x_{m, 0}} \frac{k_{i, j}\left(x_{m, l}, s, \mathbf{f}(s)\right)}{\left|x_{m, l}-s\right|^{\alpha_{j}}} d s \\
& \approx \frac{x_{1,0}}{90}\left[7\left[\frac{k_{i, j}\left(x_{m, l}, h, \mathbf{F}_{1,0}\right)}{\left|x_{m, l}-h\right|^{\alpha_{j}}}+\frac{k_{i, j}\left(x_{m, l}, 0, \mathbf{F}_{0,0}\right)}{\left|x_{m, l}-0\right|^{\alpha_{j}}}\right]+12 \frac{k_{i, j}\left(x_{m, l}, \frac{h}{2}, \mathbf{F}_{\frac{1}{2}, 0}\right)}{\left|x_{m, l}-\frac{h}{2}\right|^{\alpha_{j}}}\right. \\
& +32\left[\frac{k_{i, j}\left(x_{m, l}, \frac{h}{4}, \mathbf{F}_{\frac{1}{4}, 0}\right)}{\left|x_{m, l}-\frac{h}{4}\right|^{\alpha_{j}}}+\frac{k_{i, j}\left(x_{m, l}, \frac{3 h}{4}, \mathbf{F}_{\frac{3}{4}, 0}\right)}{\left.\left.\left|x_{m, l}-\frac{3 h}{4}\right|^{\alpha_{j}}\right]\right]+\frac{(m-1) h}{2835}\left[1 0 8 . 5 \left[\frac{k_{i, j}\left(x_{m, l}, h, \mathbf{F}_{1,0}\right)}{\left|x_{m, l}-h\right|^{\alpha_{j}}}\right.\right.}\right. \\
& \left.+\frac{k_{i, j}\left(x_{m, l}, m h, \mathbf{F}_{m, 0}\right)}{\left|x_{m, l}-m h\right|^{\alpha_{j}}}\right]+218 \frac{k_{i, j}\left(x_{m, l}, x_{\frac{1+m}{2}, 0}, \mathbf{F}_{\frac{1+m}{2}, 0}\right)}{\left|x_{m, l}-x_{\frac{1+m}{2}, 0}\right|^{\alpha_{j}}} \\
& +176\left[\frac{k_{i, j}\left(x_{m, l}, x_{\frac{3+m}{4}, 0}, \mathbf{F}_{\frac{3+m}{4}, 0}\right)}{\left|x_{m, l}-x_{\frac{3+m}{4}, 0}\right|^{\alpha_{j}}}+\frac{k_{i, j}\left(x_{m, l}, x_{\frac{1+3 m}{4}, 0}, \mathbf{F}_{\frac{1+3 m}{4}, 0}\right)}{\left|x_{m, l}-x_{\frac{1+3 m}{4}, 0}\right|^{\alpha_{j}}}\right] \\
& +512\left[\frac{k_{i, j}\left(x_{m, l}, x_{\frac{7+m}{8}, 0}, \mathbf{F}_{\frac{7+m}{8}, 0}\right)}{\left|x_{m, l}-x_{\frac{7+m}{8}, 0}\right|^{\alpha_{j}}}+\frac{k_{i, j}\left(x_{m, l}, x_{\frac{5+3 m}{8}, 0}, \mathbf{F}_{\frac{5+3 m}{8}, 0}\right)}{\left|x_{m, l}-x_{\frac{5+3 m}{8}, 0}\right|^{\alpha_{j}}}\right. \\
& +\frac{k_{i, j}\left(x_{m, l}, x_{\frac{3+5 m}{8}, 0}, \mathbf{F}_{\frac{3+5 m}{8}, 0}\right)}{\left|x_{m, l}-x_{\frac{3+5 m}{8}, 0}\right|^{\alpha_{j}}}+\frac{k_{i, j}\left(x_{m, l}, x_{\frac{1+7 m}{8}, 0}, \mathbf{F}_{\frac{1+7 m}{8}, 0}\right)}{\left.\left.\mid x_{m, l}-x_{\frac{1+7 m}{8}, 0}^{\alpha_{j}}\right]\right] .}
\end{aligned}
$$


Substituting the approximate values in (3.2), a system of $4 n$ equations with the unknowns $\mathbf{F}_{m, 1}, \mathbf{F}_{m, 2}, \mathbf{F}_{m, 3}$ and $\mathbf{F}_{m, 4}$ is obtained in each step. Starting with $\mathbf{F}_{0}=\mathbf{g}(0)$, we solve this system successively for the blocks $\left(\mathbf{F}_{0,1}, \mathbf{F}_{0,2}, \ldots, \mathbf{F}_{0,4}\right)$, $\left(\mathbf{F}_{1,1}, \mathbf{F}_{1,2}, \ldots, \mathbf{F}_{1,4}\right), \ldots$.

\subsection{Convergence analysis}

Theorem 3. The block by block method for Abel type integral equations (introduced in previous subsection) is convergent and its order of convergence is at least 5 for the functions $k_{i, j}$ and $f_{i},(i, j=1,2, \ldots, n)$ with at least 6 order continuous derivatives.

Proof. Let $\zeta=\left[\zeta_{1}, \zeta_{2}, \ldots, \zeta_{n}\right]^{T}, \beta=\left[\beta_{1}, \beta_{2}, \ldots, \beta_{n}\right]^{T}, \ldots, \eta=\left[\eta_{1}, \eta_{2}, \ldots, \eta_{n}\right]^{T}$, where $\zeta_{j}, \beta_{j}, \cdots, \eta_{j}$ are defined by (3.5). Let $\mathbf{e}_{m, l}:=\mathbf{F}_{m, l}-\mathbf{f}\left(x_{m, l}\right)$, where $\mathbf{f}\left(x_{m, l}\right) \in \mathbb{R}^{n}$ and $\mathbf{F}_{m, l} \in \mathbb{R}^{n}$ denote respectively the exact and approximate solutions of (1.1) at the point $x=x_{m, l}$. From cases I and II we can write

$$
\int_{0}^{x_{m, 0}} \mathbf{K}\left(x_{m, l}, s, \mathbf{f}(s)\right) \mathbf{H}\left(x_{m, l}, s\right) d s \approx h \sum_{i=0}^{m-1} \sum_{j=0}^{4} w_{i j} \mathbf{K}\left(x_{m, l}, x_{i, j}, \mathbf{F}_{i, j}\right) \mathbf{H}\left(x_{m, l}, x_{i, j}\right) .
$$

Then for $m=0,1, \ldots, N-1$ we have

$$
\begin{aligned}
& \left\|\mathbf{e}_{m, 1}\right\|=\| h \sum_{i=0}^{m-1} \sum_{j=0}^{4} w_{i j} \mathbf{K}\left(x_{m, 1}, x_{i, j}, \mathbf{F}_{i, j}\right) \mathbf{H}\left(x_{m, 1}, x_{i, j}\right) \\
& +\mathbf{K}\left(x_{m, 1}, x_{m, 0}, \mathbf{F}_{m, 0}\right) \zeta\left(x_{m, 1}, x_{m}, h / 4\right) \\
& +\mathbf{K}\left(x_{m, 1}, x_{m, 1}, \mathbf{F}_{m, 1}\right) \beta\left(x_{m, 1}, x_{m}, h / 4\right)+\mathbf{K}\left(x_{m, 1}, x_{m, 2}, \mathbf{F}_{m, 2}\right) \gamma\left(x_{m, 1}, x_{m}, h / 4\right) \\
& +\mathbf{K}\left(x_{m, 1}, x_{m, 3}, \mathbf{F}_{m, 3}\right) \theta\left(x_{m, 1}, x_{m}, h / 4\right)+\mathbf{K}\left(x_{m, 1}, x_{m, 4}, \mathbf{F}_{m, 4}\right) \eta\left(x_{m, 1}, x_{m}, h / 4\right) \\
& -\int_{0}^{x_{m, 0}} \mathbf{K}\left(x_{m, 1}, s, \mathbf{f}(s)\right) \mathbf{H}\left(x_{m, 1}, s\right) d s-\int_{x_{m, 0}}^{x_{m, 1}} \mathbf{K}\left(x_{m, 1}, s, \mathbf{f}(s)\right) \mathbf{H}\left(x_{m, 1}, s\right) d s \| .
\end{aligned}
$$

Similar to the proof of Theorem 1, by adding and diminishing the necessary terms and using Lipshitz conditions of the Theorem 2, we obtain

$$
\begin{aligned}
\left\|\mathbf{e}_{m, 1}\right\| & \leq h \sum_{i=0}^{m-1} \sum_{j=0}^{4} M w_{i, j} c_{i, j}\left\|\mathbf{e}_{i, j}\right\|+M\left\|\zeta\left(x_{m, 1}, x_{m}, h / 4\right)\right\|\left\|\mathbf{e}_{m, 0}\right\| \\
& +M\left\|\beta\left(x_{m, 1}, x_{m}, h / 4\right)\right\|\left\|\mathbf{e}_{m, 1}\right\|+M\left\|\gamma\left(x_{m, 1}, x_{m}, h / 4\right)\right\|\left\|\mathbf{e}_{m, 2}\right\| \\
& +M\left\|\theta\left(x_{m, 1}, x_{m}, h / 4\right)\right\|\left\|\mathbf{e}_{m, 3}\right\|+M\left\|\eta\left(x_{m, 1}, x_{m}, h / 4\right)\right\|\left\|\mathbf{e}_{m, 4}\right\| \\
& +\left\|R_{1}\right\|+\left\|R_{2}\right\|,
\end{aligned}
$$

where $R_{1}$ and $R_{2}$ are the errors of usual and product quadratures respectively. We obtain the same inequalities for $\left\|\mathbf{e}_{m, 2}\right\|,\left\|\mathbf{e}_{m, 3}\right\|$ and $\left\|\mathbf{e}_{m, 4}\right\|$.

Let again $\left\|\mathbf{e}_{m, 1}\right\|=\max _{l=1,2,3,4}\left\|\mathbf{e}_{m, l}\right\|$, then

$$
\left\|\mathbf{e}_{m, 1}\right\| \leq c h \sum_{i=0}^{m-1}\left\|\mathbf{e}_{i, 1}\right\|+h c^{\prime}\left\|\mathbf{e}_{m, 1}\right\|+\left\|R_{1}\right\|+\left\|R_{2}\right\|
$$


Therefore using the Gronwall inequality for sufficiently small $h$, yields

$$
\left\|\mathbf{e}_{m, 1}\right\| \leq \frac{\left\|R_{1}\right\|+\left\|R_{2}\right\|}{1-h c^{\prime}} e^{c x_{m} /\left(1-h c^{\prime}\right)}
$$

and so $\left\|\mathbf{e}_{m, 1}\right\| \rightarrow 0$, as $h \rightarrow 0$. For the functions $k_{i, j}$ and $f_{i},(i, j=1,2, \ldots, n)$ with at least 6 order continuous derivatives, we have $\left\|R_{1}\right\|=O\left(h^{6}\right)$ but the order of accuracy for the product integration is at least $O\left(h^{5}\right)$, since it is based on interpolation of degree 4 . Thus at the small neighborhood of $s=t$ the quadrature rule take the order $O\left(h^{5}\right)$, while out of this small neighborhood the order is $O\left(h^{6}\right)$ and so we expect the error generaly to be between five and six as the numerical results illustrate just that.

\section{Numerical results}

We consider the following examples to illustrate theoretical results of Theorems 1 and 3 and to compare numerical results of the method with the results of HPM and RBFN method.

Example 1. [8] Consider the nonlinear system

$$
\begin{aligned}
& \sin (x)-x+\int_{0}^{x}\left(f_{1}^{2}(s)+f_{2}^{2}(s)\right) d s=f_{1}(x) \\
& \cos (x)-1 / 2 \sin ^{2}(x)+\int_{0}^{x} f_{1}(s) f_{2}(s) d s=f_{2}(x)
\end{aligned}
$$

for $x \in[0,1]$, where the exact solutions are the functions $f_{1}(x)=\sin (x)$ and $f_{2}(x)=\cos (x)$.

\section{Example 2. Let}

$$
\begin{aligned}
& \mathbf{K}(x, s, \mathbf{f}(s))=\left[\begin{array}{ll}
\sin (x)-s f_{1}(s) f_{2}(s) & 0 \\
0 & s \frac{\sqrt{x}}{4} f_{1}(s)+\frac{x}{2} f_{2}(s)^{2}
\end{array}\right], \\
& \mathbf{H}(x, s)=\left[1,(x-s)^{-0.5}\right]^{T}
\end{aligned}
$$

for $x \in[0,1]$ and choose $\mathbf{g}(x)$ in such way that the exact solutions of (3.1) to be $f_{1}(x)=(1+x)^{-0.5}$ and $f_{2}(x)=x^{2}$.

Example 3. As a final example, we consider the following non-linear system of the first kind VIEs with the exact solutions $f_{1}(x)=x^{2}$ and $f_{2}(x)=x$ and $X=4$ :

$$
\mathbf{g}(x)=\int_{0}^{x} \mathbf{K}(x, s, \mathbf{f}(s)) \mathbf{H}(x, s) d s, \quad 0 \leq s \leq x \leq X
$$

where

$$
\begin{aligned}
& \mathbf{K}(x, s, \mathbf{f}(s))=\left[\begin{array}{ll}
f_{1}(s) f_{2}(s) & \left(1-x^{2}+s^{2}\right) f_{2}(s)^{3} \\
(5+x-s) f_{1}(s) & -(5+x-s) \exp \left(f_{2}(s)\right)
\end{array}\right], \\
& \mathbf{H}(x, s)=\left[(x-s)^{-1 / 3}, 1\right]^{T},
\end{aligned}
$$




$$
\mathbf{g}(x)=\left[\frac{243}{440} x^{11 / 3}-\frac{x^{6}}{12}+\frac{x^{4}}{4}, \frac{27}{220} x^{11 / 3}+\frac{27}{8} x^{8 / 3}+6+x-6 e^{x}\right]^{T} .
$$

The results in Tables $1-3$, show the absolute errors $\left(e_{F_{i}}, i=1, \ldots, n\right)$ for the examples 1-3. All results computed by programming in Maple 13.

Table 1. Numerical results of example 1.

\begin{tabular}{|c|c|c|c|c|c|c|}
\hline \multirow[t]{2}{*}{$\mathrm{x}$} & \multicolumn{2}{|c|}{ RBFN-MSHA } & \multicolumn{2}{|c|}{ HPM } & \multicolumn{2}{|c|}{ Block by Block } \\
\hline & $e_{F_{1}}$ & $e_{F_{2}}$ & $e_{F_{1}}$ & $e_{F_{2}}$ & $e_{F_{1}}$ & $e_{F_{2}}$ \\
\hline 0.1 & $8.33 \mathrm{e}-7$ & $9.47 \mathrm{e}-7$ & $3.365 \mathrm{e}-04$ & $4.864 \mathrm{e}-03$ & $1.334 \mathrm{e}-11$ & $1.741 \mathrm{e}-10$ \\
\hline 0.2 & $2.85 \mathrm{e}-7$ & $4.37 \mathrm{e}-7$ & $2.736 \mathrm{e}-03$ & $1.962 \mathrm{e}-02$ & $9.542 \mathrm{e}-11$ & $5.105 \mathrm{e}-11$ \\
\hline 0.3 & $4.35 \mathrm{e}-7$ & $7.52 \mathrm{e}-7$ & $8.950 \mathrm{e}-03$ & $4.394 \mathrm{e}-02$ & $1.179 \mathrm{e}-10$ & $2.223 \mathrm{e}-10$ \\
\hline 0.4 & $1.58 \mathrm{e}-7$ & $1.41 \mathrm{e}-7$ & $2.053 \mathrm{e}-02$ & $7.684 \mathrm{e}-02$ & $2.191 \mathrm{e}-10$ & $2.116 \mathrm{e}-10$ \\
\hline 0.5 & $5.03 \mathrm{e}-7$ & $8.75 \mathrm{e}-7$ & $4.057 \mathrm{e}-02$ & $1.174 \mathrm{e}-01$ & $2.487 \mathrm{e}-10$ & $1.912 \mathrm{e}-10$ \\
\hline 0.6 & $2.67 \mathrm{e}-7$ & $5.93 \mathrm{e}-7$ & $6.768 \mathrm{e}-02$ & $1.636 \mathrm{e}-01$ & $3.593 \mathrm{e}-10$ & $2.953 \mathrm{e}-10$ \\
\hline 0.7 & $6.69 \mathrm{e}-8$ & $6.27 \mathrm{e}-8$ & $1.052 \mathrm{e}-01$ & $2.150 \mathrm{e}-01$ & $4.132 \mathrm{e}-10$ & $2.559 \mathrm{e}-10$ \\
\hline 0.8 & $2.15 \mathrm{e}-7$ & $6.08 \mathrm{e}-7$ & $1.507 \mathrm{e}-01$ & $2.683 \mathrm{e}-01$ & $5.598 \mathrm{e}-10$ & $4.687 \mathrm{e}-10$ \\
\hline 0.9 & $1.16 \mathrm{e}-6$ & $3.25 \mathrm{e}-6$ & $2.047 \mathrm{e}-01$ & $3.224 \mathrm{e}-01$ & $2.505 \mathrm{e}-10$ & $2.355 \mathrm{e}-09$ \\
\hline 1.0 & $1.23 \mathrm{e}-6$ & $3.70 \mathrm{e}-6$ & $2.630 \mathrm{e}-01$ & $3.739 \mathrm{e}-01$ & $2.233 \mathrm{e}-11$ & $2.323 \mathrm{e}-09$ \\
\hline time & & - & & $29.593 "$ & & $1.217 "$ \\
\hline
\end{tabular}

Table 1 show the superiority of the block by block method in comparing its results (for $h=1 / 5$ ) with the results of RBFN-MshA (a modified version of Shi's algorithm) [8] and HPM [20], where the results of RBFN-MshA obtained with 6 hidden nodes and the results of HPM obtained with 4 iterations. Moreover,

- The time of computation in the block by block method is less than that in the HPM, whenever programming of both methods is done using Maple package. Also, according to the structure of HPM, increasing the number of iterations do not affect on the precision of errors.

- The values of the RBF widths affect significantly on the accuracy of results and determination of them is still a challenging problem, whereas the block by block method dose not need any starting values.

- At each step of the RBFN method, the weights are updated by using an optimization method, but the block by block method is independent of using any other method.Hence the RBFN method is more complicated than the block by block method.

Tables 2 and 3 show the numerical results for the Abel systems. Most of the available methods for solving VIEs are based on expansion of solution, for example the Taylor and Chebyshev expansion methods, Tau method, Adomian and homotopy methods and so on. These methods are efficient only for the intervals with small length (say $[0,1]$ or $[-1,1]$ ) and they are useless for the large intervals, whereas the block by block method is one of the most suitable methods for the large intervals (see table 3 for $X=4$ ). 
Table 2. Numerical results of example 2.

\begin{tabular}{|c|c|c|c|c|c|c|}
\hline \multirow[t]{2}{*}{$\mathrm{x}$} & \multicolumn{2}{|c|}{$\mathrm{N}=2$} & \multicolumn{2}{|c|}{$\mathrm{N}=4$} & \multicolumn{2}{|c|}{$\mathrm{N}=6$} \\
\hline & $e_{F_{1}}$ & $e_{F_{2}}$ & $e_{F_{1}}$ & $e_{F_{2}}$ & $e_{F_{1}}$ & $e_{F_{2}}$ \\
\hline 0.125 & $4.121 \mathrm{e}-09$ & $1.687 \mathrm{e}-08$ & $4.419 \mathrm{e}-11$ & $3.830 \mathrm{e}-11$ & $3.738 \mathrm{e}-11$ & 2.812 e-10 \\
\hline 0.25 & $5.267 \mathrm{e}-09$ & $9.049 \mathrm{e}-09$ & $3.437 \mathrm{e}-09$ & $9.796 \mathrm{e}-09$ & $2.443 \mathrm{e}-11$ & 2.131 e-10 \\
\hline 0.375 & $3.666 \mathrm{e}-08$ & $1.455 \mathrm{e}-07$ & $3.243 \mathrm{e}-09$ & $1.881 \mathrm{e}-09$ & $2.126 \mathrm{e}-11$ & 4.649 e-10 \\
\hline 0.5 & $2.338 \mathrm{e}-07$ & $3.501 \mathrm{e}-07$ & $4.095 \mathrm{e}-09$ & $6.061 \mathrm{e}-09$ & $3.202 \mathrm{e}-11$ & 5.847 e- 10 \\
\hline 0.625 & $1.954 \mathrm{e}-07$ & $1.871 \mathrm{e}-07$ & $1.714 \mathrm{e}-10$ & $1.951 \mathrm{e}-09$ & $1.576 \mathrm{e}-10$ & 3.887 e-10 \\
\hline 0.75 & $1.917 \mathrm{e}-07$ & $1.288 \mathrm{e}-07$ & $1.593 \mathrm{e}-10$ & $4.378 \mathrm{e}-09$ & $5.484 \mathrm{e}-09$ & 8.121 e-10 \\
\hline 0.875 & $1.468 \mathrm{e}-07$ & $2.546 \mathrm{e}-07$ & $2.058 \mathrm{e}-09$ & $3.527 \mathrm{e}-09$ & $1.709 \mathrm{e}-09$ & $1.661 \mathrm{e}-09$ \\
\hline 1 & $1.600 \mathrm{e}-07$ & $4.611 \mathrm{e}-07$ & $1.837 \mathrm{e}-09$ & 7.773e-09 & $1.442 \mathrm{e}-09$ & 2.705 e-09 \\
\hline
\end{tabular}

Table 3. Numerical results of example 3.

\begin{tabular}{cccccc}
\hline \multirow{2}{*}{$\mathrm{x}$} & \multicolumn{3}{c}{$\mathrm{N}=5$} & & \multicolumn{2}{c}{$\mathrm{N}=10$} \\
\cline { 2 - 3 } \cline { 5 - 6 } & $e_{F_{1}}$ & $e_{F_{2}}$ & & $e_{F_{1}}$ & $e_{F_{2}}$ \\
\hline 0.2 & $1.029 \mathrm{e}-10$ & $7.869 \mathrm{e}-11$ & & $3.695 \mathrm{e}-12$ & $1.523 \mathrm{e}-12$ \\
0.4 & $3.943 \mathrm{e}-10$ & $6.647 \mathrm{e}-10$ & & $4.970 \mathrm{e}-11$ & $7.022 \mathrm{e}-11$ \\
1 & $1.328 \mathrm{e}-08$ & $7.855 \mathrm{e}-09$ & & $1.567 \mathrm{e}-11$ & $2.064 \mathrm{e}-11$ \\
1.4 & $4.470 \mathrm{e}-10$ & $1.208 \mathrm{e}-09$ & & $2.495 \mathrm{e}-11$ & $2.275 \mathrm{e}-10$ \\
2 & $7.214 \mathrm{e}-09$ & $7.545 \mathrm{e}-09$ & & $2.458 \mathrm{e}-09$ & $6.868 \mathrm{e}-10$ \\
2.4 & $2.190 \mathrm{e}-09$ & $6.225 \mathrm{e}-08$ & & $5.472 \mathrm{e}-10$ & $1.675 \mathrm{e}-10$ \\
2.8 & $1.047 \mathrm{e}-08$ & $1.342 \mathrm{e}-08$ & & $1.716 \mathrm{e}-10$ & $3.168 \mathrm{e}-09$ \\
3 & $5.491 \mathrm{e}-08$ & $7.842 \mathrm{e}-09$ & & $1.674 \mathrm{e}-09$ & $1.444 \mathrm{e}-09$ \\
3.4 & $1.941 \mathrm{e}-07$ & $1.162 \mathrm{e}-07$ & & $4.776 \mathrm{e}-09$ & $1.000 \mathrm{e}-09$ \\
4 & $3.036 \mathrm{e}-07$ & $1.208 \mathrm{e}-07$ & & $7.769 \mathrm{e}-09$ & $1.485 \mathrm{e}-09$ \\
\hline
\end{tabular}

\section{References}

[1] J. Abdalkhani. A numerical approach to the solution of Abel integral equations of the second kind with nonsmooth solution. Journal of Computational and Applied Mathematics, 29(3):249-255, 1990. http://dx.doi.org/10.1016/03770427(90)90011-N.

[2] A. Akyüz-Daşcioğlu. Chebyshev polynomial solutions of systems of linear integral equations. Applied Mathematics and Computation, 151(1):221-232, 2004. http://dx.doi.org/10.1016/S0096-3003(03)00334-5.

[3] J. Biazar, E.Babolian and R. Islam. Solution of a system of Volterra integral equations of the first kind by Adomian method. Applied Mathematics and Computation, 139(2-3):249-258, 2003. http://dx.doi.org/10.1016/S00963003(02)00173-X.

[4] H. Brunner. The numerical solution of integral equations with weakly singular kernels. Num. Anal., 1066:50-71, 1984. http://dx.doi.org/10.1007/BFb0099518.

[5] H. Brunner and H.J.J.te Riele. Volterra-type integral equations of the second kind with nonsmooth solutions. Integral Equations, 6:187-203, 1984.

[6] K. Diethelm, J.M. Ford, N.J. Ford and M. Weilbeer. Pitfalls in fast numerical solvers for fractional differential equations. Jour- 
nal of Computational and Applied Mathematics, 186(2):482-503, 2006. http://dx.doi.org/10.1016/j.cam.2005.03.023.

[7] M.T. Giraudo and L. Sacerdote. First entrance time distribution millimolarity in a model neuron. Quaderni di Dipartimento di Mathematica Universitá di Torino, 18, 2002.

[8] A. Golbabai, M. Mammadov and S. Seifollahi. Solving a system of nonlinear integral equations by an RBF network. Computer $\&$ Mathematics with Applications, 57(10):1651-1658, 2009. http://dx.doi.org/10.1016/j.camwa.2009.03.038.

[9] R. Gorenflo and S. Vessella. Abel Integral Equations: Analysis and Applications. Springer, Berlin, 1991. http://dx.doi.org/10.1007/BFb0084665.

[10] B. Jumarhon and S. Mckee. On the heat equation with nonlinear and nonlocal boundary conditions. Journal of Mathematical Analysis and Applications, 190:806-820, 1995. http://dx.doi.org/10.1006/jmaa.1995.1113.

[11] R. Katani and S. Shahmorad. Block by block method for the systems of nonlinear Volterra integral equations. Applied Mathematical Modelling, 34:400-406, 2010. http://dx.doi.org/10.1016/j.apm.2009.04.013.

[12] K. Maleknejad and M. Shahrezaee. Using Runge-Kutta method for numerical solution of the system of Volterra integral equation. Appl. Math. and Comp., 149(2):399-410, 2004. http://dx.doi.org/10.1016/S0096-3003(03)00148-6.

[13] K. Maleknejad and A. Salimi Shamloo. Numerical solution of singular Volterra integral equations system of convolution type by using operational matrices. Applied Mathematics and Computation, 195(2):500-505, 2008. http://dx.doi.org/10.1016/j.amc.2007.05.001.

[14] S. McKee, T. Tang and T. Diogo. An Euler-type method for two-dimensional Volterra integral equations of the first kind. IMA Journal of Numerical Analysis, 20(3):423-440, 2000. http://dx.doi.org/10.1093/imanum/20.3.423.

[15] R. Metzler and J. Klafter. The random walk's guide to anomalous diffusion: a fractional dynamics approach. Physics Reports, 339(1):1-77, 2000. http://dx.doi.org/10.1016/S0370-1573(00)00070-3.

[16] M. Rabbani, K. Maleknejad and N. Aghazadeh. Numerical computational solution of the Volterra integral equations system of the second kind by using an expansion method. Applied Mathematics and Computation, 187(2):1143-1146, 2007. http://dx.doi.org/10.1016/j.amc.2006.09.012.

[17] L. Tao and H. Yong. Extrapolation method for solving weakly singular nonlinear Volterra integral equations of the second kind. Journal of Mathematical Analysis and Applications, 324(1):225-237, 2006.

http://dx.doi.org/10.1016/j.jmaa.2005.12.013.

[18] M. E. A. El Tom. Application of spline functions to system of Volterra integral equation of the first and second kinds. IMA, Appl. Math., 17(3):295-310, 1976.

[19] A. Young. The application of approximate product-integration to the numerical solution of integral equations. Proceeding of Royal Society of London. Series A, 224:561-573, 1954. http://dx.doi.org/10.1098/rspa.1954.0180.

[20] E. Yusufoğlu. A homotopy perturbation algorithm to solve a system of Fredholm-Volterra type integral equations. Mathematical and Computer Modelling, 47:1099-1107, 2008. 